\title{
Atomic resolved material displacement on graphite surfaces by scanning tunnelling microscopy
}

Philip Moriarty and Greg Hughes

Physics Department, Dublin City Universily, Glasnevin, Dublin 9, Ireland

(Received 16 December 1991; accepted for publication 27 February 1992)

\begin{abstract}
Atomic scale modifications and subsequent atomic resolution imaging has been achieved on the highly oriented pyrolytic graphite surface in air. Application of short pulse voltages, above a minimum threshold voltage of $3.5 \mathrm{~V}$, across the tunneling gap results in the displacement of a layer or more of atoms to form a hole and create a neighboring mound or "nanodot" from the displaced atoms. We have found a correlation between the hole and "nanodot" volume at the atomic level and observe an asymmetric displacement of material in all cases of feature creation. Nanofeatures as small as four carbon atoms at beta sites have been created. Our experimental observations are consistent with the modification process depending on the gradient in the electric field induced by the rise time of the bias pulse voltage and not the pulse duration. Interesting faceting behavior has also been observed around some hole edges. Tip bias pulsing sometimes induced a tip, and not a surface modification, resulting in a change in the observed tunneling image.
\end{abstract}

The scanning tunnelling microscope (STM), has been shown to be capable of providing real space images with atomic resolution on a variety of surfaces in a range of environments. It is being increasingly used as a tool to modify surfaces at the nanometer scale. A wide range of lithographic and surface modification techniques using the STM's ability to generate high electric fields between the tip and the surface have been demonstrated in a number of experiments. Molecular manipulation ${ }^{1}$ and nanometer scale hole formation ${ }^{2}$ on graphite have both been previously reported, the former presenting a $4 \AA$ area benzene ring pinned to three beta site atoms. Clusters of $\mathrm{Ag}$ and $\mathrm{Au}$ surface deposits on graphite have been imaged with atomic resolution ${ }^{3}$ and recently, nanometer scale structures as small as $1 \mathrm{~nm}$, have been fabricated on the same surface using a STM in the presence of trimethylaluminum. ${ }^{4}$ Surface diffusion appreciably distorts the features created by tip bias induced modifications on gold, ${ }^{5-7}$ over a time scale of minutes. In this letter, we present the results of a study aimed at understanding the surface modification process on graphite by imaging the features created at atomic scale resolution.

The tunneling microscope used in these experiments is the commercially available Nanoscope II from Digital Instruments, CA, and the mechanically sharpened Pt-Ir tips were from the same supplier. Samples of highly oriented pyrolytic graphite (HOPG) from Agar Scientific, Essex, UK, were cleaved immediately before imaging. All images in this letter were obtained in the constant height mode (nanoscope current mode) with a positive sample bias voltage of $25 \mathrm{mV}$ and a set point tunneling current of $1 \mathrm{nA}$. Voltage pulses of various duration and magnitude, ranging from 12 to $512 \mu \mathrm{s}$ and -10 to $+10 \mathrm{~V}$ were applied across the tunneling gap during an interruption in the scanning routine which was software controlled. The threshold voltage for feature creation was found to depend on scan area at a fixed scan frequency, however, no dependence of feature size on pulse duration, within the above limits was detected. A pulse of $500 \mu$ s duration was subsequently used for all experiments. The tip condition was found to play a critical role in the lithographic process. The success rate in producing atomic scale modifications varied between $0 \%$ and $90 \%$ from tip to tip.

In order to minimize thermal drift during the experiments, the STM was allowed to scan for a period of $2 \mathrm{~h}$ before any surface modification was attempted. A zoom on a typical feature crealed by the application of a $5 \mathrm{~V}, 500 \mu \mathrm{s}$ pulse in an original scan area of $10 \times 10 \mathrm{~nm}^{2}$ is shown in Fig. 1. All the features created under these conditions consist of a mound of raised material (a nanodot) displaced to one side of the accompanying hole. The smallest feature we have been able to create in a scan area of $2.5 \times 2.5 \mathrm{~nm}^{2}$ is shown in Fig. 2(a). A schematic drawing of the surface atomic arrangement following the bias pulse, which is illustrated in Fig. 2(b), shows that this modification involves the displacement of four atoms at beta sites to form a nanodot and hole, each four atomic sites in area. This suggests that the hole volume can be directly correlated with the nanodot volume. Although the finite radius of the tip will have a strong influence on the apparent hole depth, a single layer modification seems to be present. In order to fabricate features of this size the highest possible tip resolution was necessary, however, even fulfilling condition modifications as small as these were by no means routine, with about $10 \%$ success rate. Being able to accurately analyze this small feature in terms of a direct correlation between hole size and displaced material would lead us to believe that the larger features created in the larger scan areas can be similarly correlated.

The threshold voltage for hole formation was found to increase from $3.5 \mathrm{~V}$ for a scan arca less than $20 \times 20 \mathrm{~nm}^{2}$, to $8 \mathrm{~V}$ for an area of $25 \times 25 \mathrm{~nm}^{2}$ at the same scan frequency $(40 \mathrm{~Hz}$ ). A bove this scan area we have been unable to create any holes. When this is coupled with the fact that hole size does not depend on pulse duration, but on a scan area up to $25 \times 25 \mathrm{~nm}^{2}$, it suggests that the critical factor in 


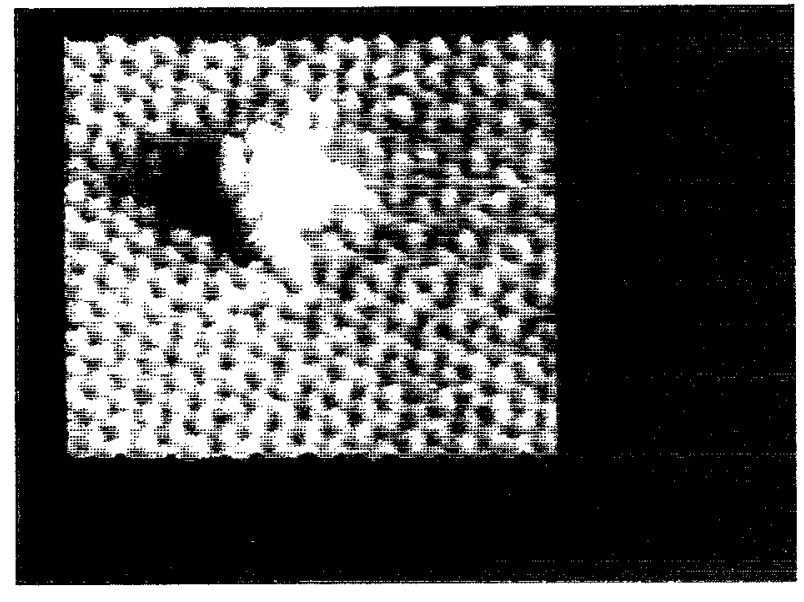

FIG. 1. A zoom of a typical feature displayed in a $4 \times 4 \mathrm{~nm}^{2}$ area, created by the application of a $5 \mathrm{~V}, 500 \mu \mathrm{s}$ pulse in an original scan area of $10 \times 10$ $\mathrm{nm}^{2}$. A nanodot with a neighboring faceted hole in the graphite lattice.

the creation of these features is the rate of change in the electric field gradient between the tip and the surface atons. It is the rise time of the bias pulse voltage above a threshold value, and not the pulse duration, which appears to determine the formation of these surface features. One
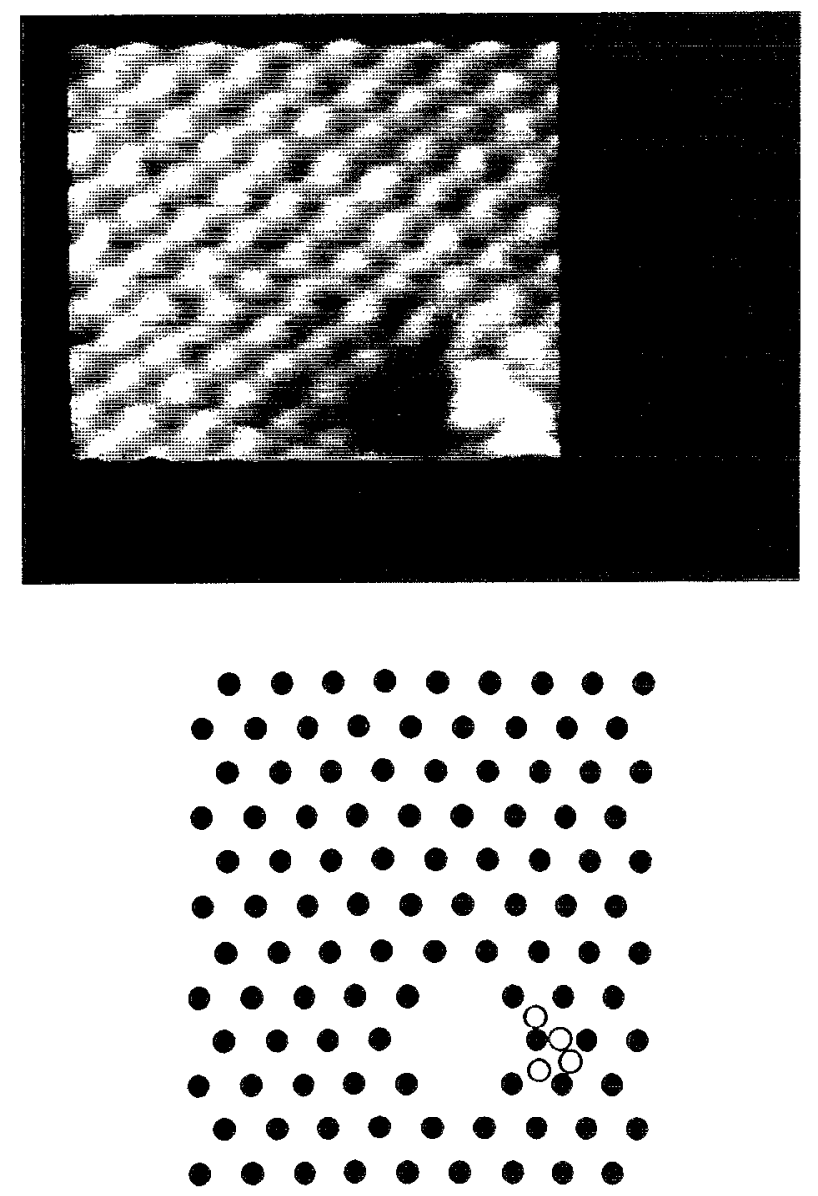

FIG. 2. An image of the smallest hole created displayed in a scan area of $2.5 \times 2.5 \mathrm{~nm}^{2}$ is shown in (a), while (b) is a schematic drawing of the resulting atomic surface arrangement with only atoms at beta sites shown and the open circles representing the displaced atoms. way of diluting the field gradient experienced by individual surface atoms is to increase the scan area while maintaining the scan frequency. The inability to create holes above a scan area of $25 \times 25 \mathrm{~nm}^{2}$ suggests that while the pulse is being applied, the tip velocity is such that the electric field gradient is not sufficiently localized over individual atoms to cause surface disruption. A similar effect is observed if a smaller surface area $\left(20 \times 20 \mathrm{~nm}^{2}\right)$, is scanned at a frequency higher than $80 \mathrm{~Hz}$. This would again be consistent with the tip being in motion when the bias pulse is applied. Therefore, our results would suggest that a critical factor in the surface modification process is the gradient in the electric field induced by the rise time of the bias pulse in a spatially localized area. The threshold voltage for hole formation therefore scales with the effective scan speed.

The asymmetric displacement of material clearly seen in Fig. 1 was not observed in a similar study on graphite reported by Albrecht et al., ${ }^{2}$ although the overall size and shape of the created features are comparable. We can eliminate the possibility of physical contact between the tip and surface during the pulse sequence by placing constraints on the tip motion and we do not believe it can be attributed to asymmetry in the tip geometry. While we do not as yet understand the reason for this asymmetry, it does have an interesting side effect which can be observed in Fig. 1. There is clear evidence of faceting behavior around the hole edges where no excess material has been deposited. A sharp $60^{\circ}$ angle corresponding to alignment of the atoms along crystallographic axes was observed in approximately $60 \%$ of the created holes. This faceting appears to be instantaneous on the time scale of the imaging. Attempts to follow the evolution of the faceted features over a 30-60 s time period immediately following pulse application produced little discernible change. This contrasts with a recent STM assisted surface etching study ${ }^{8}$ on a quasi-twodimensional conducting oxide which noted a change of the fabricated pits with time, to form faceted features. However, the formation of similar triangular shaped structures on tungsten diselenide by pulse biasing has recently been reported ${ }^{9}$ where the defects were observed to grow in size for times up to $1 \mathrm{~h}$. In our investigations on graphite, all modifications were found to be extremely stable and were imaged for periods of hours with no observable change.

During the course of the modification process described it was found that applying a pulse in some cases resulted not in a surface modification, but in a change in the STM image (Fig. 3). It has been previously shown by Mizes et al. ${ }^{10}$ that the experimental images for a layered material are dominated by only three independent Fourier components and that an asymmetric tip consisting of not one but two outermost atoms, cause the variety of images observed in this single figure. We conjecture, that the applied pulse in these cases leads not to a surface modification but to a tip modification causing the interference (due to double imaging) of the individual Fourier components.

In summary, we have succeeded in modifying the graphite surface at the atomic level to form features down to four atoms in area. The surface modification, which involves the physical displacement of atoms, appears to 


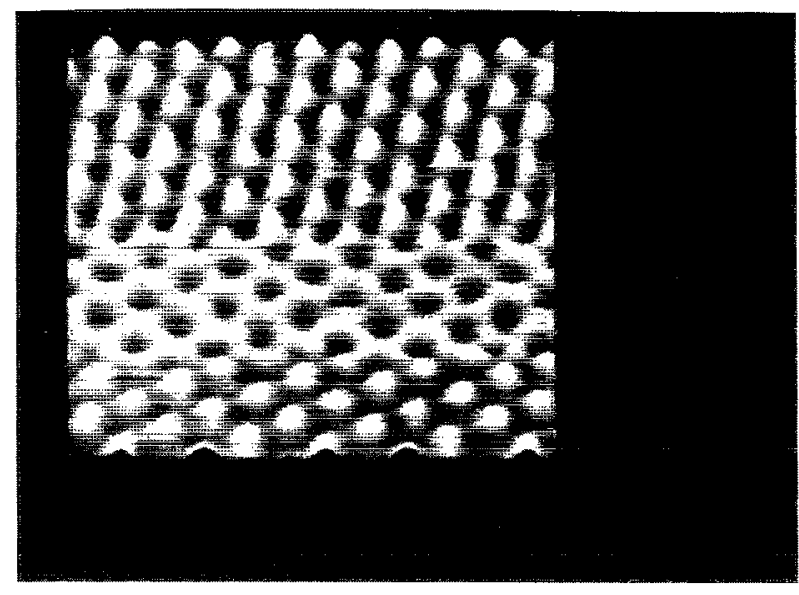

FIG. 3. A variety of surface images resulting in the bias pulse causing a tip modification rather than a surface modification, displayed in a 2.5 $\times 2.5 \mathrm{~nm}^{2}$ scan arca.

depend on the rate of change of a spatially localized electric field which is related to the rise time of the bias pulse. While the exact modification process is not well understood, rearrangement of surface atoms stimulated by the high field gradients involved in the pulsing sequence appear to play a critical role in the formation of these features.

We would like to thank the National Metrology Laboratory in The Irish Science and Technology Agency (EOLAS) for providing the facilities to carry out this work and one of us, P. M. acknowledges financial support from EOLAS under the Applied Research Award Scheme.

${ }^{1}$ J. S. Foster, J. E. Frommer, and P. C. Arnett, Nature 331, 324 (1988).

${ }^{2}$ T. R. Albrecht, M. M. Dovek, M. D. Kirk, C. A. Lang, C. F. Quate, and D. P. E. Smith, Appl. Phys. Lett. 55, 1727 (1989).

${ }^{3}$ D. W. Abraham, K. Sattler, E. Ganz, H. J. Manin, E. Thompson, and J. Clarke, Appl. Phys. Lett. 49, 853 (1986).

${ }^{4}$ S-T. Yau, D. Saltz, A. Wriekat, and M. H. Hayfeh, J. Appl. Phys. 69, 2970 (1991).

${ }^{5}$ Y. Z. Li, L. Vazquez, R. Piner, R. P. Andes, and Reifenberger, Appl. Phys. Lett. 54, 1424 (1989).

${ }^{6}$ J. Schneir, R. Sonnenfeld, O. Marti, P. K. Hansma, J. E. Demuth, and R. J. Hamers, J. Appl. Phys. 63, 717 (1988).

${ }^{7}$ L. Nagahara, S. M. Lindsay, T. Thundat, and U. Knipping, J. Microse. 152, 145 (1988).

${ }^{8}$ D. Saulys, G. Rudd, and E. Garfunkel, J. Appl. Phys. 69, 1707 (1991).

${ }^{9}$ S. Akari, R. Moller, and K. Dransfeld, Appl. Phys. Lett. 59, 243 (1991).

${ }^{10}$ H. A. Mizes, S.-I. Park and W. A. Harrison, Phys. Rev. B 36, 4491 (1987). 